\title{
Robust Stabilization OF A MAin IrRigation CANAL POOL VIA FRACTIONAL ORDER PI CONTROLLER: A GRAPHICAL INVESTIGATION
}

\author{
Radek Matušu \\ Centre for Security, Information and Advanced Technologies (CEBIA - Tech) \\ Faculty of Applied Informatics, Tomas Bata University in Zlin \\ nám. T. G. Masaryka 5555, 76001 Zlín, Czech Republic
}

\begin{abstract}
This contribution is focused on graphical investigation of robust stability for a feedback control loop containing the parametrically uncertain second order time-delay model of a main irrigation canal pool and a fractional order PI controller. The robust stability of the family of fractional order closed-loop characteristic quasipolynomials is analyzed by means of the sampled value sets and application of the zero exclusion condition.
\end{abstract}

Keywords: Robust stability analysis; fractional order control; time-delay systems; PI controller; parametric uncertainty.
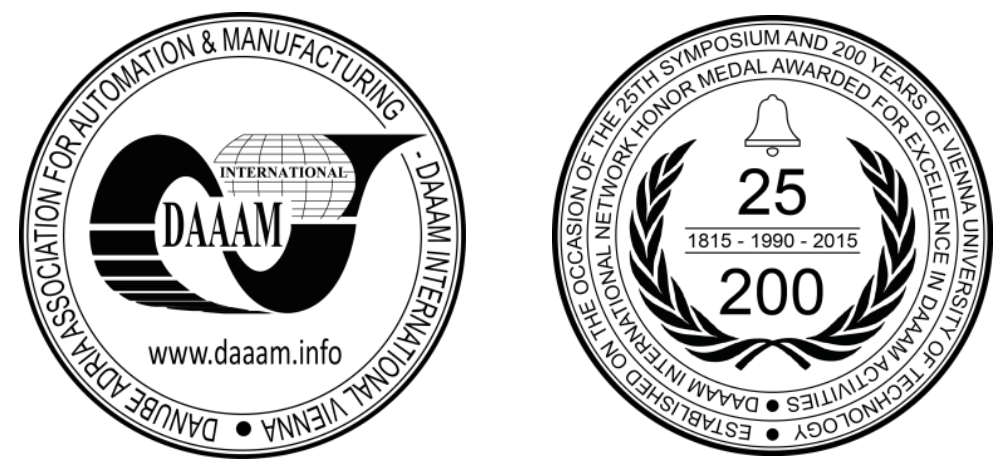

This Publication has to be referred as: Matusu, R[adek] (2016). Robust Stabilization of a Main Irrigation Canal Pool via Fractional Order PI Controller: A Graphical Investigation, Proceedings of the 26th DAAAM International Symposium, pp.0212-0216, B. Katalinic (Ed.), Published by DAAAM International, ISBN 978-3-902734-07-5, ISSN 1726-9679, Vienna, Austria DOI:10.2507/26th.daaam.proceedings.029 


\section{Introduction}

The fractional order calculus and its utilization in control theory belong among the highly attractive contemporary research topics [1-3]. However, fractional order control has not only theoretical meaning, but it had already influenced also many real control applications. The nice examples of the practical use with direct impact on more effective management of precious water resources were published in [4-9], where the main irrigation canals were controller under various conditions.

Systems with parametric uncertainty represent common but also effective way of incorporating the uncertainty into mathematical model. Consequently, they allow relatively easy description of originally much more complicated, nonlinear or varying real-life systems by means of linear time-invariant (LTI) models. In such systems, the structure (model order) is supposed to be known and fixed but the parameters of the model are bounded somehow. Frequently, they are considered just to vary within given real intervals. One of the most important tasks is to analyze the robust stability of the control loops affected by parametric uncertainty, i.e. the stability under all possible variations of uncertain parameters has to be verified. Quite naturally, the combination of the robust stability problem under parametric uncertainty with fractional order systems has become also the important research subject in the recent years [10-13].

This contribution focuses on graphical analysis of robust stability for a feedback control loop containing the parametrically uncertain second order time-delay model of a main irrigation canal pool and a fractional order PI controller [4]. The robust stability of the family of fractional order closed-loop characteristic quasipolynomials is analyzed via the sampled value sets and application of the zero exclusion condition [14]. The similar investigation of robust stability for a main irrigation canal pool with different mathematical model and integer order PID or fractional order PI controller can be found in [15].

\section{Main irrigation canal pool - mathematical description and controller design}

According to [4-9], a typical main irrigation canal consists of several pools separated by undershot gates that are used for regulating the water distribution from one pool to the next one. The controlled variables are water levels at the end of the canal pool and the manipulated variables are the gate positions. The canal pool dynamic behaviour can be described by the second order time-delay system, i.e. by transfer function [4]:

$$
G\left(s, K, T_{1}, T_{2}, \Theta\right)=\frac{K}{\left(T_{1} s+1\right)\left(T_{2} s+1\right)} e^{-\Theta s}
$$

where $K$ represents a static gain, $T_{1}$ and $T_{2}$ are time constants, and $\Theta$ stands for time-delay. The first time constant $T_{1}$ is the dominant one and corresponds to the dynamics of the canal pool while the second shorter time constant $T_{2}$ is associated to the motors and gates dynamics. The nominal values of the parameters (related to the normal hydraulic operation regime) are $K_{0}=0.0401, T_{10}=880.79[s], T_{20}=81.27[s]$ and $\Theta_{0}=360[s]$. Nevertheless, since the discharge through the upstream gates can vary in some operational range, the parameters of the model (1) are consequently supposed to lie within given intervals [4]:

$$
\begin{aligned}
& K \in[0.01,0.1] \\
& T_{1} \in[500,15000] \\
& T_{2} \in[10,300] \\
& \Theta \in[300,360]
\end{aligned}
$$

As can be seen, the parameter variations are really wide and finding a suitable controller which will assure robust stabilization (or moreover also a specified minimum level of performance) for the assumed model is a non-trivial task. In the paper [4], the following fractional order PI controller was designed:

$$
C(s)=0.021 \frac{1+155 s^{0.8}}{s}=\frac{0.021+3.255 s^{0.8}}{s}
$$

and compared with a classical integer order PI controller. The robust stability of the closed control loop with considered parametrically uncertain second order time-delay model (1) and recommended fractional order PI controller (3) will be analyzed by means of the graphical method described in the following Section.

\section{Robust stability analysis for (fractional order time-delay) systems with parametric uncertainty}

As the stability of LTI systems can be investigated via the stability of its characteristic polynomials, the primary object of interest from the robust stability viewpoint is the family of closed-loop characteristic polynomials. 
Besides, if the controlled plant model contains time delay term, the family of closed-loop characteristic quasipolynomials has to be analyzed.

In this work, the very universal graphical method for robust stability investigation is utilized. It consists of combination of the value set concept with the zero exclusion condition [14].

The family of polynomials can be denoted as [14]:

$$
P=\{p(\cdot, q): q \in Q\}
$$

where $q$ is a vector of uncertain parameters (uncertainty) confined by some uncertainty bounding set $Q$ (which is frequently a multidimensional box).

The value set at frequency $\omega \in \mathfrak{R}$ is given by [14]:

$$
p(j \omega, Q)=\{p(j \omega, q): q \in Q\}
$$

The zero exclusion condition for Hurwitz stability of family of continuous-time polynomials (4) says [14]: Assume invariant degree of polynomials in the family, pathwise connected uncertainty bounding set $Q$, continuous coefficient functions $\rho_{i}(q)$ for $i=0,1,2, \ldots, n$ and at least one stable member $p\left(s, q^{0}\right)$. Then the family $P$ is robustly stable if and only if the complex plane origin is excluded from the value set $p(j \omega, Q)$ at all frequencies $\omega \geq 0$, that is $P$ is robustly stable if and only if:

$0 \notin p(j \omega, Q) \quad \forall \omega \geq 0$

More details can be found in [14] or other related literature. Furthermore, the principle of the value set concept and the zero exclusion condition is valid also for more general value sets (e.g. for the closed-loop uncertain quasipolynomials in which the plant numerator contains the time-delay term). polynomials.

Besides, the works [10-13] extended the idea of the value set concept also to fractional order uncertain

In this paper, the value sets are plotted for a closed-loop characteristic quasipolynomial (loop with time-delay plant family (1) and fractional order PI controller (3)) and its visualization is based on sampling the uncertain parameters and on computation of partial points of the value sets for a considered frequency range. Thanks to the applied sampling (brute-force) method, the value sets of the quasipolynomial can be easily computed and consequently the robust stability can be analyzed with the assistance of standard zero exclusion condition.

\section{Example - robust stability analysis}

The goal of the example is to verify if the fractional order PI controller (3) robustly stabilizes the plant family (1), i.e. if it guarantees the stability of the feedback control loop for all possible variations of parameters (2). The family of fractional order closed-loop characteristic quasipolynomials is:

$$
p_{C L}\left(s, K, T_{1}, T_{2}, \Theta\right)=\left(T_{1} s+1\right)\left(T_{2} s+1\right) s+K e^{-\Theta s}\left(3.255 s^{0.8}+0.021\right)
$$

where uncertain parameters can lie within intervals (2). The value sets are computed for the frequency range from 0 to 0.004 with the step 0.00008 while for the each frequency the variations of uncertain parameters are constructed by means of sampling:

$$
\begin{aligned}
& K=0.01: 0.005: 0.1 \\
& T_{1}=500: 500: 15000 \\
& T_{2}=10: 10: 300 \\
& \Theta=300: 10: 360
\end{aligned}
$$

The calculated value sets are visualized in Fig. 1 and the zoomed version of the same value sets (in order to see better the situation near the complex plane origin) is plotted in Fig. 2. 


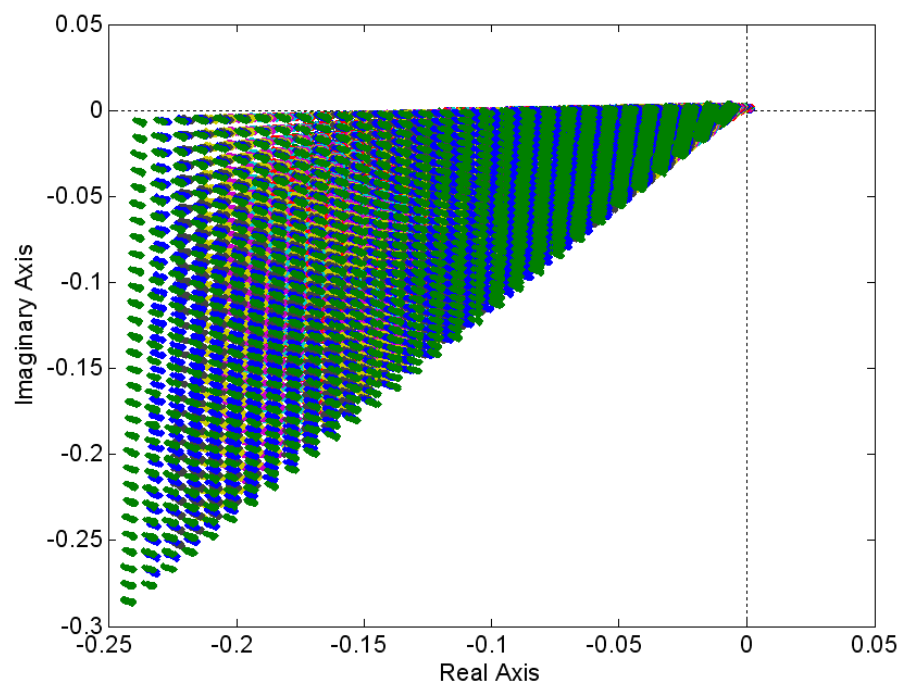

Fig. 1. Value sets for family of fractional order closed-loop characteristic quasipolynomials (7) - full view.

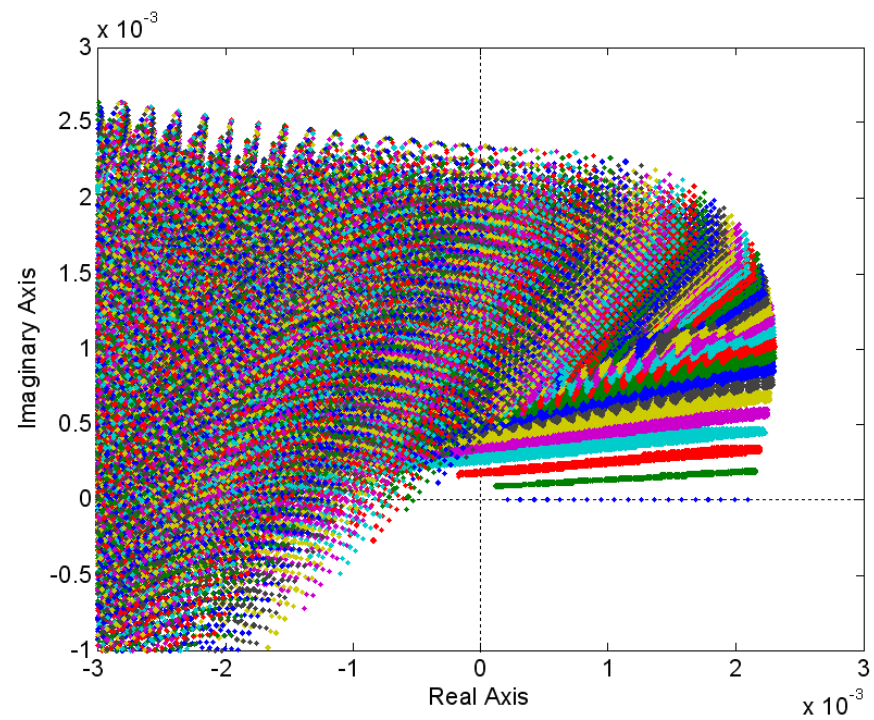

Fig. 2. Value sets for family of fractional order closed-loop characteristic quasipolynomials (7) - zoomed view.

As can be easily verified, the family (7) definitely contains a stable member. Moreover, Fig. 2 clearly demonstrates that the origin of the complex plane (zero point) is excluded from the value sets. It means the closed control loop is robustly stable (i.e. stable for all possible variations of canal pool model parameters) with designed fractional order PI controller (3).

\section{Conclusion}

The contribution has been aimed to graphical method of robust stability analysis for a feedback control loop containing a main irrigation canal pool modelled as the second order time-delay plant with parametric uncertainty and a fractional order PI controller. The robust stability of the obtained family of fractional order closed-loop characteristic quasipolynomials is investigated through the depiction of the sampled value sets and direct application of the zero exclusion condition.

\section{Acknowledgements}

This work was supported by the Ministry of Education, Youth and Sports of the Czech Republic within the National Sustainability Programme project No. LO1303 (MSMT-7778/2014). 


\section{References}

[1] Y. Chen, I. Petráš, D. Xue, Fractional Order Control - A Tutorial, In: Proceedings of the 2009 American Control Conference, St. Louis, MO, USA, 2009.

[2] R. E. Gutiérrez, J. M. Rosário, J. A. T. Machado, Fractional Order Calculus: Basic Concepts and Engineering Applications, Mathematical Problems in Engineering, Vol. 2010 (2010), 19 p.

[3] I. Podlubný, Fractional-Order Systems and PI $\lambda \mathrm{D} \mu$-Controllers, IEEE Transactions on Automatic Control, Vol. 44, No. 1 (1999), pp. 208-214.

[4] V. Feliu-Batlle, R. Rivas-Pérez, L. Sánchez-Rodríguez, F. J. Castillo-García, A. Linarez-Saez, Robust Fractional Order PI Controller for a Main Irrigation Canal Pool, In: Proceedings of the 17th IFAC World Congress, Seoul, Korea, 2008.

[5] V. Feliu-Batlle, R. Rivas-Pérez, F. J. Castillo-García, Fractional Robust Control to Delay Changes in Main Irrigation Canals, In: Proceedings of the 16th IFAC World Congress, Prague, Czech Republic, 2005.

[6] V. Feliu-Batlle, R. Rivas-Pérez, L. Sánchez-Rodríguez, Fractional robust control of main irrigation canals with variable dynamic parameters, Control Engineering Practice, Vol. 15, No. 6 (2007), pp. 673-686.

[7] V. Feliu-Batlle, R. Rivas-Pérez, F. J. Castillo-García, L. Sánchez-Rodríguez, Smith predictor based robust fractional order control: Application to water distribution in a main irrigation canal pool, Journal of Process Control, Vol. 19, No. 3 (2009), pp. 506-519.

[8] V. Feliu-Batlle, R. Rivas-Pérez, F. J. Castillo-García, Fractional order controller robust to time delay variations for water distribution in an irrigation main canal pool, Computers and Electronics in Agriculture, Vol. 69, No. 2 (2009), pp. 185-197.

[9] V. Feliu-Batlle, R. Rivas-Pérez, F. J. Castillo-García, L. Sánchez-Rodríguez, A. Linarez-Saez, Robust fractional order controller for irrigation main canal pools with time-varying dynamical parameters, Computers and Electronics in Agriculture, Vol. 76, No. 2 (2011), pp. 205-217.

[10] N. Tan, Ö. F. Özgüven, M. M. Özyetkin, Robust stability analysis of fractional order interval polynomials, ISA Transactions, Vol. 48, No. 2 (2009), pp. 166-172.

[11] B. Şenol, C. Yeroğlu, Robust Stability Analysis of Fractional Order Uncertain Polynomials, In: Proceedings of the 5th IFAC Workshop on Fractional Differentiation and its Applications, Nanjing, China, 2012.

[12] B. Şenol, C. Yeroğlu, Computation of the Value Set of Fractional Order Uncertain Polynomials: A 2q Convex Parpolygonal Approach, In: Proceedings of the 2012 IEEE International Conference on Control Applications, Dubrovnik, Croatia, 2012.

[13] C. Yeroğlu, B. Şenol, Investigation of robust stability of fractional order multilinear affine systems: 2q-convex parpolygon approach, Systems \& Control Letters, Vol. 62, No. 10 (2013), pp. 845-855.

[14] B. R. Barmish, New Tools for Robustness of Linear Systems, Macmillan, New York, USA, 1994.

[15] R. Matušů, R. Prokop, Robust Stability of Fractional Order Time-Delay Control Systems: A Graphical Approach, Mathematical Problems in Engineering, Vol. 2015 (2015), 9 p. 